\title{
Interoception: The Eighth Sensory System: Practical Solutions for Improving Self-Regulation, Self-Awareness and Social Understanding of Individuals with Autism Spectrum and Related Disorders
}

\author{
K. J. Mahler: Shawnee Mission KS, AAPC, 2015, 186 pp, \$29.95 (paper), ISBN 978-1- \\ 942197-14-0
}

\author{
Punit Shah ${ }^{1}$ (I) \\ Published online: 25 June 2016 \\ (C) The Author(s) 2016. This article is published with open access at Springerlink.com
}

Interoception: The Eighth Sensory System is an interesting book written by an author with her finger on the pulse of autism research. Kelly Mahler, an occupational therapist specializing in Autism Spectrum Disorders (ASD), provides an accessible introduction to interoception (the ability to perceive the internal state of one's body) and its association with social-emotional abilities such as empathy and emotional regulation. This captures the idea that sensing internal bodily changes is related to the ability to understand and regulate oneself, and thereby understand and interact with other people (Chapter 1). This is followed by an explanation of why impaired interoception seemingly underlies many difficulties in ASD with a view to help autistic individuals understand their condition (Chapter 2). Drawing on her experience as a school-based therapist, Mahler includes a series of instruments to measure interoceptive ability (Chapter 3) and 'how-to' exercises to improve interoception for managing socio-emotional problems (Chapter 4). Interesting vignettes about autistic individuals are interspersed throughout the book, highlighting daily difficulties which may be due to interoceptive failure (e.g., emotional outbursts), including strategies for how such problems can be controlled. The book finishes with an incisive conclusion about why interoception is important for understanding ASD (Chapter 5).

Interoception has been neglected by psychologists and rarely been addressed directly in autism research. The book is

Punit Shah

punit.shah@kcl.ac.uk

1 MRC Social, Genetic and Developmental Psychiatry Centre, Institute of Psychiatry, Psychology and Neuroscience, King's College London, University of London, London SE5 8AF, UK therefore timely, novel, and it has attracted positive buyer reviews. It is supported by a complimentary foreword written by Professor A.D. (Bud) Craig who is the world's foremost expert on interoception. Like Craig, I was impressed with Mahler's grasp of contemporary research, some of which has been conducted by my group leader and his collaborators (see Bird and Cook 2013). The book contains many hypotheses that are currently under investigation, reinforcing hopes that our research holds translational value. The text is very wellwritten and transforms complex concepts - usually found in neurobiological research articles-into an accessible format. Taken together, it has potential for researchers, autistic individuals, parents and therapists to better understand and manage ASD. Because of growing interest in the clinical relevance of interoception, and the books intuitive format, it is expected that Mahler's ideas may be adopted by researchers and clinicians alike. However, this may be premature and there are some caveats that warrant discussion.

First, the author does not mention research showing that interoception was, using a measure of cardiac perception, found to be similar in children with and without ASD (Schauder et al. 2015). While Mahler cites research on impaired interoception in autistic adults that was published after Schauder et al.'s (2015) study, it is unfortunate this important research was ignored given that the book is built on the premise that interoception is impaired in children with ASD. There may be reasons underlying Schauder and colleagues' result (lack of appropriate control tasks or because autism and control groups were matched for alexithymia perchance; see below), however these issues were not discussed. The reader should therefore be aware that cardiac perception (as found in in the book for measuring and training interoception) in children with ASD may be comparable to typical children, and also that there is currently no evidence that interoception can be improved 
in children. Furthermore, although interoceptive training is an exciting prospect, even if it is shown to be possible in children as found in adults, it remains to be determined whether or not it leads to transferable changes in socialemotional functioning as Mahler suggests.

Second, the author has misunderstood the co-occurrence of ASD and alexithymia. Alexithymia is characterized by difficulties in recognizing emotions from internal bodily sensations. The author correctly notes that alexithymia frequently co-occurs with ASD (approximately $55 \%$; see Bird and Cook 2013) and lists alexithymia questionnaires under measures by which to assess interoception (Chapter 3). Unfortunately, however, Mahler does not recognize that ASD and alexithymia are independent constructs: Alexithymia is not required for a diagnosis of ASD nor it is present in all individuals with autism. Equally, many individuals in the neurotypical population (about 10\%) have elevated degrees of alexithymia with no ASD. Mahler therefore mistakes research on socio-emotional atypicalities due to alexithymia after controlling for autism (see Bird and Cook 2013) for evidence of impaired interoception in ASD. It was also not made clear that the relationship between alexithymia and impaired cardiac perception (e.g., Herbert et al. 2011) is unrelated to ASD. Instead, research findings on atypical interoception that Mahler cites to support her ideas, are likely due to the fact that ASD groups in these studies contained more alexithymic individuals than the control groups, misleading her to an erroneous link between autism and impaired interoception. Indeed, Brewer et al. (2015) have argued that, where observed in ASD, signs of abnormal emotion processing and impaired interoception are likely due to co-occurring alexithymia. This was confirmed in a recent study in adults, which showed that autism was not associated with interoceptive accuracy after controlling for co-occurring alexithymia and other clinical traits (Shah et al. 2016). Mahler will not have been aware of the most recent research at the time of writing the book. However, the impact of co-occurring alexithymia in ASD, together with recent research, calls into question the conclusions and therapeutic implications arising from the book. As such, the book requires revision and needs development pending further empirical investigation. I agree with Mahler that interoceptive training may be therapeutic for ASD and other disorders, but potentially only for individuals with co-occurring alexithymia. Alternatively, it is possible that alexithymic individuals have such diminished interoception that training programs may not be effective. Or cognitive impairments (e.g., attentional atypicalities) in individuals with both ASD and alexithymia could render interoception interventions ineffective. In addition, it is possible that interoceptive training has to be administered during critical periods in childhood in order to minimize interoceptive difficulties in adulthood. All of these possibilities require rigorous investigation before interoception research can be properly translated into clinical practice. Until then, it is suggested that interoception should not be measured or trained in autistic individuals without consideration of cooccurring alexithymia, for example using a questionnaire such as the 20-item Toronto Alexithymia Scale (Bagby et al. 1994; see www.bit.ly/TAS-20).

In summary, Mahler's well-written book will introduce both researchers and non-researchers to the concept of interoception; its relationship with social-emotional abilities and its therapeutic potential. I encourage the readership of the Journal of Autism and Developmental Disorders to peruse the book, which they will find informative and enjoyable. However, there are concerns about the evidence presented in support of the book, particularly given the confusion over the co-occurrence of ASD and alexithymia. It is concluded that, although it is an interesting addition to the growing literature on autism and interoception, the book requires refinement if interoceptive training is going to reach its full (therapeutic) potential.

Acknowledgments P.S. is supported by a studentship from the Medical Research Council. I thank Geoffrey Bird and Lucy Livingston for looking at earlier drafts of the manuscript.

Open Access This article is distributed under the terms of the Creative Commons Attribution 4.0 International License (http://creative commons.org/licenses/by/4.0/), which permits unrestricted use, distribution, and reproduction in any medium, provided you give appropriate credit to the original author(s) and the source, provide a link to the Creative Commons license, and indicate if changes were made.

\section{References}

Bagby, R. M., Parker, J. D., \& Taylor, G. J. (1994). The twenty-item Toronto Alexithymia Scale-I. Item selection and cross-validation of the factor structure. Journal of Psychosomatic Research, $38(1), 23-32$.

Bird, G., \& Cook, R. (2013). Mixed emotions: The contribution of alexithymia to the emotional symptoms of autism. Translational Psychiatry, 3(7), e285. doi:10.1038/tp.2013.61.

Brewer, R., Happé, F., Cook, R., \& Bird, G. (2015). Commentary on "Autism, oxytocin and interoception": Alexithymia, not Autism Spectrum Disorders, is the consequence of interoceptive failure. Neuroscience and Biobehavioral Reviews, 56, 348-353. doi:10. 1016/j.neubiorev.2015.07.006.

Herbert, B. M., Herbert, C., \& Pollatos, O. (2011). On the relationship between interoceptive awareness and alexithymia: Is interoceptive awareness related to emotional awareness? Journal of Personality, 79(5), 1149-1175. doi:10.1111/j.1467-6494.2011. 00717.x.

Schauder, K. B., Mash, L. E., Bryant, L. K., \& Cascio, C. J. (2015). Interoceptive ability and body awareness in autism spectrum disorder. Journal of Experimental Child Psychology, 131, 193-200. doi:10.1016/j.jecp.2014.11.002.

Shah, P., Hall, R., Catmur, C., \& Bird, G. (2016). Alexithymia, not autism, is associated with impaired interoception. Cortex. doi:10. 1016/j.cortex.2016.03.021. 